\title{
The effect of short-term and long-term application of fisetin on experimentally induced airway hyperreactivity
}

${ }^{1}$ Comenius University in Bratislava, Jessenius Faculty of Medicine in Martin Biomedical Center Martin, Martin, Slovak Republic

${ }^{2}$ Comenius University in Bratislava, Jessenius Faculty of Medicine in Martin Department of Pharmacology, Martin, Slovak Republic

Received 27 February, 2017, accepted 24 March, 2017

Abstract Background: Fisetin, a derivate from the flavonol group may possess a variety of pharmacological effects. The aim of the presented study was to evaluate the bronchodilatory effect of fisetin after the acute or the chronic administration to guinea pigs with allergic airway inflammation.

Methods: Experimental animals were sensitized and challenged by ovalbumin. Fisetin was administered in dose $5 \mathrm{mg} / \mathrm{kg} / \mathrm{p}$.o., either once after the end of 21-days sensitization or daily during the 21-days sensitization. By using the whole-body plethysmograph, we monitored the specific airway resistance, a parameter of airway hyperreactivity in vivo. The changes of the specific airway resistance were evaluated after the short-term inhalation of the bronchoconstriction mediator -histamine $\left(10^{-6} \mathrm{~mol} .1^{-1}\right)$.

Results: Our results showed that the short-term as well as the long-term administration of fisetin caused decrease of the specific airway resistance values. The bronchodilatory effect of fisetin was comparable to the long-acting beta, sympathomimetic salmeterol after the long-term administration. The measurements of the bronchodilatory activity after single administration have revealed more prolonged effect of fisetin comparing to the short-acting beta ${ }_{2}$ sympathomimetic - salbutamol, as this remained even after the 5 hours, when salbutamol was already ineffective.

Conclusion: In conclusion, flavonol - fisetin has shown bronchodilatory potential. In the light of this fact, fisetin may represent potential substance that can be effective in both prevention as well as control of airway inflammation symptoms.

Keywords Airway hyperreactivity-fisetin-airway inflammation

\section{INTRODUCTION}

The airway hyperreactivity is one of the main attribute of allergic asthma. It is defined as an exaggerated contraction response of the airways to different kind of endogenous and exogenous stimuli (Bossé, 2012). It is believed, that airway inflammation plays a central role in the development of airway hyperreactivity. Airway infiltration by inflammatory cells can impair the integrity of the bronchial epithelium. Due to this change, the release of bronchodilating substances is decreased and the formation of bronchoconstriction active kinins is augmented. Moreover, inflammatory cells such as eosinophils and mast cell release the mediators with the capacity to cause bronchial smooth muscle contraction and exudation of plasma, resulting in the thickening of airway wall and the modification of mechanical properties of airway smooth muscle (O’Byrne \& Inman, 2003; Fredberg, 2004).

There are evidences, that flavonoids possess bronchodilatory effect and inhibit the synthesis of pro-inflammatory cytokines and the release of chemical mediators in allergic asthma (Tanaka \& Takahashi, 2013). In the light of this fact, the aim of the presented study was to estimate the effect of shortterm and long-term application of flavonol derivate fisetin on airway hyperreactivity. The bronchodilatory activity of fisetin was evaluated on experimental model of allergic airway inflammation in guinea pigs. This model simulates the development of airway hyperreactivity (Franova et al., 2013). The ability of fisetin to relax airway smooth muscle was 
compared with the effect of clinically used bronchodilator drugs. The short-acting beta ${ }_{2}$-symphatomimetic salbutamol was used to compare the acute bronchodilatory effect of fisetin and the long-acting beta ${ }_{2}$-symphatomimetic salmeterol as a reference drug for the chronic bronchodilatory effect.

\section{MATERIAL AND METHODS}

All processes were approved by the Institutional Ethic Committee of the Jessenius Faculty of Medicine in Martin, Slovakia (permission IRB 00005636) and the experiment procedures were carried out in accordance with the Slovakian and European legislation (decision No. EK 1178/2012). Male guinea pigs (TRIK, 200-350g) were used in the experiment. The animals were obtained from the Department of Toxicology and the Laboratory of Animal Breeding, Dobra Voda, Bratislava, Slovakia (SK CH 24016), and located in animal house of Biomedical Center Martin JFM CU for one week of quarantine; food and water were available ad libitum with a standard air conditioning system.

Material and chemicals: Fisetin, salbutamol, salmeterol, histamine and ovalbumin (OVA, egg albumin grade III) were purchased from Sigma-Aldrich Chemicals (St. Louis, MO, USA).

Experimental design: In both experimental procedures (acute and chronic therapy), the animals were divided into the following groups: i) the healthy control group, ii) the negative control groups - animals sensitized with ovalbumin, iii) the positive control groups - animals sensitized with ovalbumin and treated by the reference drugs - the acute therapy salbutamol ( $4 \mathrm{mM}$ once by inhalation for $5 \mathrm{~min}$ ), the chronic therapy - salmeterol $(0.17 \mathrm{mM}$ daily by inhalation for $5 \mathrm{~min})$, iv) the therapeutic groups- animals sensitized with ovalbumin and treated by fisetin ( $5 \mathrm{mg} / \mathrm{kg} / \mathrm{p}$.o.). Each group consisted of 10 guinea pigs. A model of allergic asthma on guinea pigs by chronic ovalbumin challenge was used in the experiment (Franova et al., 2013). During the acute therapy, the effect of single administration of fisetin was evaluated after the end of 21-day sensitization and the changes in the specific airway resistance were recorded before, 1 and $5 \mathrm{~h}$ after application of tested substances. During the chronic therapy, the animals received fisetin daily during the 21 -days sensitization. The measurements were performed $24 \mathrm{~h}$ after both the last allergen exposure and treatment.

The evaluation of airway reactivity in vivo: In vivo airway reactivity was evaluated using a double chamber whole-body plethysmograph (Rodents Double-Chamber Plethysmograph, Hugo Sachs Electronik-Harvard Apparatus, type 855 with HSE Pulmodyn Pennock W software). The changes in the specific airway resistance after a short-term $(2 \mathrm{~min}$ ) inhalation of bronchoconstriction mediator - histamine $\left(10^{-6} \mathrm{~mol}^{-\mathrm{I}^{-1}}\right)$ were considered as an indicator of airway reactivity in vivo. There was an interval of $1 \mathrm{~min}$ between histamine exposure and the airway resistance measurement. During this interval, fresh air was insufflated into the breathing chamber.
Statistics: The statistical analysis was performed using ANOVA test followed by Bonferroni post hoc test. Differences were considered statistically significant when $\mathrm{P}$-values were below 0.05 . All results are expressed as the means \pm SEM.

\section{RESULTS}

Repetitive exposure of guinea pigs to ovalbumin caused significant increase of the specific airway resistance compared with the healthy control group. The single dose of fisetin induced the highly significant decrease of the specific airway resistance values. In contrast to the effect of salbutamol, fisetin proved its bronchodilatory activity $1 \mathrm{~h}$ as well as $5 \mathrm{~h}$ after the acute therapy (Fig. 1).

During chronic therapy, the 21-day sensitization of animals by ovalbumin resulted in non-significant increase of the specific airway resistance. Although the long-term administration of fisetin caused a decrease in the specific airway resistance after histamine nebulization compared to negative control group, this effect was not statistically relevant. The similar changes without statistical significance were monitored in animal group treated by reference drug salmeterol (Fig. 2).

\section{DISCUSSION}

Fisetin (3, 3', 4', 7- tetrahydroxyflavone), a flavonoid is mostly discussed in literature sources for its various spectra of pharmacological properties. Several experimental studies have showed its anti-inflammatory, anti-oxidant activities, cardioprotective, neuroprotective and anticancer properties (Khan et al., 2013). Moreover, fisetin belongs to the group of flavonols, that exhibit the well-known bronchodilatory activity (Ko, 2003). Flavonol derivates such as quercetin, kaempferol and morin proved the ability to suppress allergen-induced airway hyperreactivity and airway infiltration by inflammatory cells. Gong etal. (2012) showed that kaempferol administration suppressed eosinophil infiltration of the airways, which are the source of bronchoconstriction active substances. Recent studies in allergy confirmed that quercetin is more effective

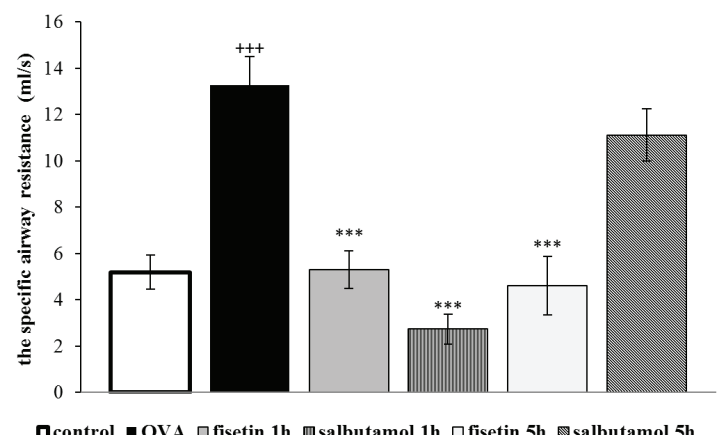

Figure 1. The specific airway resistance values after the acute administration of fisetin.Data are expressed as the means \pm SEM; statistical significance $+++P<0.001$ control vs. OVA, ${ }^{* * *} P<0.001$ tested substances vs. OVA. 


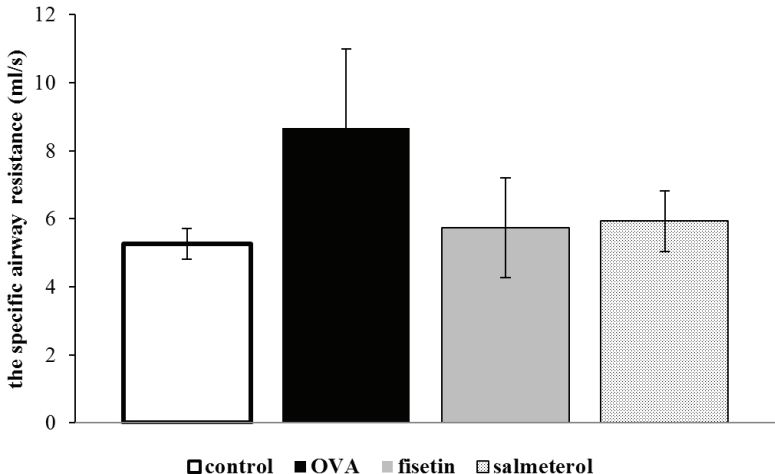

Figure 2. The specific airway resistance values after the chronic administration of fisetin. Data are expressed as the means \pm SEM.

in the stabilization of mast cells than Cromolyn sodium (Weng et al., 2012). Franova et al. (2016) found out, that the bronchodilatory activity of morin was higher than the effect of salmeterol.

In current study, we studied the bronchodilatory effect of other flavonol derivate fisetin under in vivo conditions. Our results confirmed the ability of fisetin to relax airway smooth muscle, which correlate with the findings of previously mentioned studies. The 21-day application of fisetin decreased the airway reactivity comparing to treatment naïve animals with allergen induced airway inflammation. However these results were not statistically significant, observed decrease in specific airway resistance was similar to that of reference drug salmeterol. We assume that the mentioned tendency of fisetin to reduce airway smooth muscle contraction after long-term administration of fisetin is a result of its suppressive effect on airway inflammation, which was demonstrated in the study of Goh et.al. (2012).

Regardless the anti-inflammatory activity, fisetin was able to attenuate the bronchoconstriction response even after single administration, when the anti-inflammatory impact of fisetin should be minimal. Therefore it is highly probable, that fisetin can act directly on the mechanisms regulating the bronchoconstriction. Indeed recent study has demonstrated that fisetin inhibits phospholipase $C \beta$ and phosphodiesterase$4 D 3$, the enzymes which play the important role in maintenance of airway smooth muscle contraction (Brown et al., 2016)

\section{CONCLUSIONS}

In conclusion, according to the above-mentioned results, we can say, that the present study demonstrated the beneficial effect of fisetin on the airway hyperreactivity related to allergic inflammation. We believe, that our findings may contribute to the development of drugs useful in prevention or in the treatment of airway inflammatory diseases such as asthma.

\section{ACKNOWLEDGEMENTS}

This work was supported by the project „Biomedical Center Martin" ITMS code: 26220220187 , the project is co-financed by EC sources; the Slovak Research and Development Agency under the contract No. APVV-0305-12; CEKR I; CEKR II; Grant VEGA No. 1/0165/14 and Grant MZ 201/35-UKMA-12.

\section{References}

[1] Bossé Y. Asthmatic airway hyperresponsiveness: the ants in the tree. Trends in Molecular Medicine. 2012; 18: 627-633.

[2] Brown A, Danielsson J, Townsend EA, et al. Attenuation of airway smooth muscle contractility via flavonol-mediated inhibition of phospholipase-C $\beta$. Am J Physiol Lung Cell Mol Physiol. 2016; 310:L747-L758.

[3] Franova S, Joskova M, Sadlonova V, Pavelcikova D, Mesarosova L, Novakova E, Sutovska M. Experimentalmodel of allergic asthma. Advances in Experimental Medicine and Biology. 2013; 756: 4955.

[4] Franova S, Kazimierova I, Pappova L, Joskova M, Plank L, Sutovska M. Bronchodilatory, antitussive and anti-inflammatory effect of morin in the setting of experimentally induced allergic asthma. Journal of Pharmacy and Pharmacology. 2016; 68(8):1064-1072.

[5] Fredberg JJ. Bronchospasms and its biophysical basis in airway smooth muscle. In Respiratory Research. 2004; 5: 2.

[6] Goh FY, Upton N, Guan S, et al. Fisetin, a bioactive flavonol, attenuates allergic airway inflammation through negative regulation of NF-KB. European Journal of Pharmacology. 2012; 679: 109-116.
[7] Gong JH, Shin D, Han SY, Kim JL, Kang YH. Kaempferol suppresses eosinophil infiltration and airway inflammation in airway epithelial cells and in mice with allergic asthma. J Nutr. 2012; 142: 47-56.

[8] Khan N, Syed DN, Ahmad N, Mukhtar H. Fisetin: A dietary antioxidant for health promotion. Antioxidant Redox Signaling. 2013; 19(2): 151-162.

[9] Ko WCH, Liu PY, Chen JL, Leu IJ, Shin CM. Relaxant effects of flavonoids in isolated in guinea pig trachea and their structure activity relationship. In Planta Medica. 2003; 12: 1086-1090.

[10] O’Byrne PM, Inman MD. Airway Hyperresponsiveness. Chest Journal. 2003; 123: 411-416.

[11] Tanaka T, Takahashi R. Flavonoids and asthma. Nutrients. 2013; 5: 2128-2143.

[12] Weng Z, Zhang B, Asadi S, et al. Quercetin is more effective than cromolyn in blocking human mast cellcytokine release and inhibits contact dermatitis and photosensitivity in humans. PLoSOne. 2012; 7: 33805. 